\title{
BLUEBERRY INHIBITS LPS-INDUCED MURINE MICROGLIA CELL ACTIVATION AND CELL DEATH
}

\author{
M. A. Miah ${ }^{1 *}$, S. S. Choi ${ }^{2}$ and S. H. Lee ${ }^{2}$ \\ ${ }^{1}$ Department of Physiology, Bangladesh Agricultural University, Myemensingh-2202, Bangladesh; ${ }^{2}$ School of \\ Life Sciences and Biotechnology, Korea University, Seoul, Korea
}

\begin{abstract}
Microglia activation plays a pivotal role in varying stress conditions including oxidation, inflammation and certain neurodegenerative diseases. iNOS and PrxI are known to up regulate in LPS-activated microglia in vitro. Natural fruits including various berries containing both antioxidants and anti-inflammatory polyphenols may attenuate such stress conditions. We investigated the effects of blueberry extracts (BBE) on the LPS-activated stress related genes up regulation using BV-2 microglia cells in vitro. BV-2 cells were cultured in DMEM with $10 \%$ FBS, were pre-incubated with $50 \mu \mathrm{g} / \mathrm{ml}$ BBE for $0,1 \mathrm{~h}$ and $12 \mathrm{~h}$, and treated with $1 \mu \mathrm{g} / \mathrm{ml}$ LPS for $24 \mathrm{~h}$. The levels of mRNA and protein expression showed significant differences in iNOS and PrxI among the treatment groups. Hoechst and PI staining showed that BBE protects cells from activation induced death. Intracellular levels of ROS were increased by LPS stimulation over the level in the control cells whereas BBE treatment significantly lowered the LPS stimulated ROS levels at a time dependent manner. High expressions of iNOS and PrxI are indication of microglia activation and treatment with BBE in LPS-activated BV-2 cells cause down regulation of stress-related genes expression and protect the cells from over activation and death.
\end{abstract}

Key words: Microglia, blueberry extract, LPS, iNOS, PrxI

\section{INTRODUCTION}

Neuroinflammation is largely mediated by activated microglial cells. Microglia are primarily involved in immune surveillance (Davalos et al., 2005; Nimmerjahn et al., 2005) but when activated show macrophage-like capabilities including phagocytosis, inflammatory cytokine production and antigen presentation (Garden and Moller, 2006).The inflammatory agent lipopolysaccharide (LPS) present on the outer membrane of all Gramnegative bacteria and is a strong activator of host defense responses, such as increased reactive oxygen species (ROS) production, and the expression of a variety of proinflammatory cytokines and proteins, such as inducible nitric oxide synthase (iNOS) (Palsson et al., 2004). It causes cell activation and death.

ROS are generated in biological systems as free radicals and removed by the antioxidant system in the body. When the level of free radicals exceeds the ability of the antioxidant system, lipid peroxidation and DNA and protein degradation occur, which result in aging and various diseases including inflammation, cancer, Parkinson's disease, cardiovascular diseases (Halliwell B,1994).

Peroxiredoxins (Prxs) are a family of antioxidant proteins that have been linked to the prevention of oxidative stress and inflammation. Previous studies shown that PrxI was up regulated in LPS activated microglia. Microglial PrxI expression is highly dependent on the degree of microglial activation and useful marker for microglia activation (Kim et al., 2008).

Recent study shown that supplementation with fruits and vegetables rich in polyphenolic is beneficial in both forestalling and reversing the deleterious effects of aging on neuronal communication and behavior (Joseph et al., 2005). The observed protection may be due to the antioxidant and anti-inflammatory properties of the polyphenolic compounds found in fruits and vegetables (Rice-Evans and Miller, 1996). Blueberries have the highest antioxidant capacity among all fruits and vegetables as measured by the oxygen radical absorbance capacity assay (Prior and Cao, 2000, Gordillo et. al., 2009). Blueberries and other polyphenolic-enriched fruits and vegetables have been shown to possess a variety of beneficial properties in protection against inflammation, carcinogenesis, and chronic diseases (Yi et al., 2005).

*Corresponding e-mail address: alam.dina@gmail.com

Copyright (c) 2012 Bangladesh Society for Veterinary Medicine

All rights reserved 0250/2012 


\section{A. Miah and others}

As activated microglia are supposed to cause or exacerbate several neurodegenerative diseases, pharmacological strategies to suppress microglial activity are being explored as therapies. Studies of natural antioxidants are attractive to investigators as therapeutic purposes. Recent study indicated that treatments with blueberry extract inhibited the production of the inflammatory mediator nitric oxide (NO) as well as the cytokines interleukin- $1 \beta$ and tumor necrosis factor- $\alpha$ in cell conditioned media from LPS activated BV-2 microglia (Lau et al., 2007), but the role of blueberry on stress related genes remain unclear. The present study was investigated to know the effects of blueberry on LPS activated genes regulations and activation induced microglia cell death.

\section{MATERIALS AND METHODS}

\section{BV-2 microglia cell culture}

Dulbecco's modified eagle's medium (DMEM) and fetal bovine serum (FBS) were obtained from WelGENE Inc. (South Korea), penicillin and streptomycin from Sigma (St. Louis, MO). Cell culture dishes and multiwell plates were purchased from SPL life Sciences (South Korea) and Corning incorporated (NY, USA) respectively. LPS (E. coli 055:B5) obtained from Calbiochem (Germany). BV-2 cell line is an immortalized murine microglia and were maintained and propagated by DMEM supplemented with $10 \% \mathrm{FBS}$ and antibiotics at $37^{\circ} \mathrm{C}$ in a humified incubator under $5 \% \mathrm{CO}_{2}$ (Reis et al., 2006).

\section{BBE preparation and treatment}

BB extracts were prepared as described by Lau et al. (2007) with some modifications. Briefly, intact blueberry were mixed and grinded, centrifuged at $2500 \mathrm{rpm}$ for $20 \mathrm{~min}$, supernatant were transferred to tubes, then again centrifuged at $25000 \mathrm{rpm}$ for $1 \mathrm{~h}$ at $4^{0} \mathrm{C}$. The upper portion was transferred to new eppendorf tube and stored at $80^{\circ} \mathrm{C}$ until using. Media supplemented with BBE $(50 \mathrm{ug} / \mathrm{ml})$ were prepared immediately before the experiments. RNA isolation and semiquantitative RT- PCR analysis

BV-2 cells at $2 \times 10^{5}$ per $60 \mathrm{~mm}$ culture dish were cultured in DMEM with $10 \%$ FBS for $24 \mathrm{~h}$, then cells were preincubated with $50 \mu \mathrm{g} / \mathrm{ml} \mathrm{BBE}$ for 0 (no preincubation), 1 and $12 \mathrm{~h}$ and treated with $1 \mu \mathrm{g} / \mathrm{ml} \mathrm{LPS}$ for $24 \mathrm{~h}$. Total RNA was isolated from lysed cells using Trizol® (Invitrogen). For reverse transcription, contaminating DNA in RNA solution was digested by I U of RQ1 DNAse (Promega, Madison, WI, USA) as recommendation of manufacturer's instructions. The first strand cDNA was synthesized from $1 \mu \mathrm{g}$ of DNAse treated total RNA using Oligo dT in a volume of $20 \mu \mathrm{l}$ at $37^{\circ} \mathrm{C}$ for $45 \mathrm{~min}$ and $95^{\circ} \mathrm{C}$ for $5 \mathrm{~min}$. the first strand cDNA $(2 \mu \mathrm{l})$ was used for PCR amplification in a $50 \mu \mathrm{l}$ reaction mixture. PCR was performed using the following primers:

iNOS- Sense: 5'CCCTTCCGAAGTTTCTGGCAGCAGC-3, Antisense- 5'GGCTGTCAGAGCCTCGTGGCTTTGG-3

PrxI- Sense- 5'-TGGCTCGACCCTGCTGATAGCAAG-3, Antisense- 5'-CTGAAAGAGATACCTTCATCAG-3

G3PDH- Sense- 5'TTCACCACCATGGAGAAGGC-3 Antisense: 5'GGCATGGACTGTGGTCATGA-3

\section{Gel electrophoresis and Western blot}

Cell culture conditions and treatments were same as described in the previous section. For Western blot analysis, $30 \mu \mathrm{g}$ of total cell extracts was prepared and separated using $10 \%$ sodium dodecyl sulfatepolyacrylamide gels (SDS-PAGE) and transferred to nitrocellulose membrane. The membranes were incubated for $1 \mathrm{~h}$ with $5 \%$ skim milk in TBS containing $0.02 \%$ Tween 20 (TBST) to avoid nonspecific binding. Then they were incubated with polyclonal rabbit anti-Prxl (Alexis biochemical, USA), monoclonal mouse iNOS (abcam, USA), and anti-rabbit GAPDH antibodies. The membrane was subsequently incubated with AP conjugated antirabbit, anti-rat and anti-mouse IgG. The immunoreactivity bands were detected using color detection solution containing NBT and BCIP.

\section{Intracellular ROS detection by flow cytometer}

ROS accumulation in the cell was detected by 5-(6-) chloromethyl-2', 7'dichlorodihydrofluorescein diacetate (CM- $\mathrm{H}_{2}$ DCFDA; molecular probes, Eugene) according to manufacturer and procedures described by Reis $e$ a $a l$. (2006.). Briefly, BV-2 cells were cultured in 6 well dishes, preincubated with BBE followed by $1 \mu \mathrm{g} / \mathrm{ml}$ of LPS for $18 \mathrm{~h}$ then labelled with $800 \mathrm{nM} \mathrm{CM}-\mathrm{H}_{2} \mathrm{DCFDA}$ for $20 \mathrm{~min}$. at $37^{\circ} \mathrm{C}$ in a humidified atmosphere of $5 \% \mathrm{CO}_{2}$ in incubator, the cells were washed and re-suspended in PBS and analyzed immediately by flow cytometer, using Cell Quest software (Becton Dickinson, Mountain View, CA). 
Cell death assay by Hoechst-Propidium Iodide (PI) staining

Cell death was assayed by Hoechst and PI staining according the methods by Roy et al.(2005). BV-2 cells were treated with BBE and LPS for $24 \mathrm{~h}$ in 12 well tissue culture plates. Both medium containing floating cells and trypsin-EDTA dissociated cells were transferred to $15 \mathrm{ml}$ conical tube. Cells were washed twice with medium by centrifugation and incubated with equal volume of Hoechst-PI solution for $20 \mathrm{~min}$ at room temperature. Cells were again washed twice with fresh medium by centrifugation. Cell pellets were suspended in $10 \mu \mathrm{l}$ of medium, and transferred onto the glass slide and seen under fluorescence microscope.

\section{RESULTS AND DISCUSSION}

\section{BBE attenuated expressions of iNOS and PrxI at a time dependent manner}

To know the effect of BBE on iNOS and PrxI expression in LPS activated microglia, we measured the mRNA and protein levels of iNOS and PrxI by semiquantative RT-PCR and Western blot (Figure 1). The mRNA levels of iNOS and PrxI were minimal in untreated cell but were increased in LPS treated cells. Pretreatment with BBE significantly down regulated the LPS induced increased expressions of iNOS and PrxI at a time dependent manner. iNOS and PrxI protein level also found as similar pattern detected by Western blot. The inhibitory property of BBE was found more effective in cells pretreated with BBE for $12 \mathrm{~h}$.

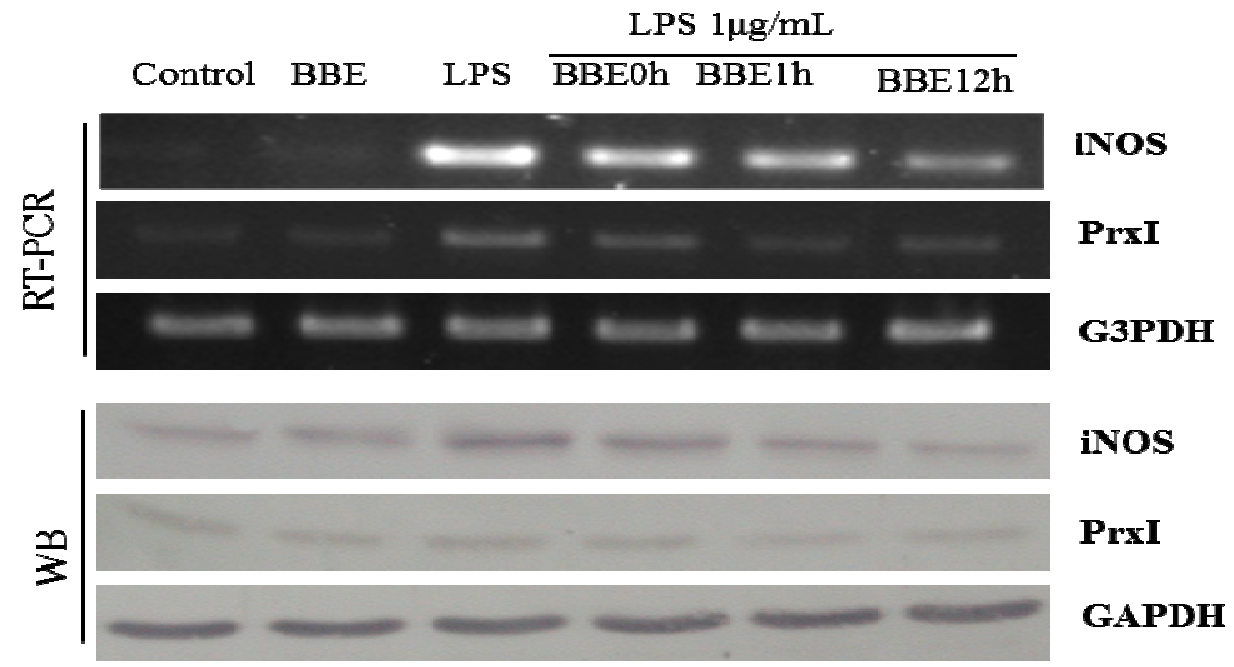

Figure 1. Effect of BBE on expressions of iNOS and PrxI. BV-2 cells were pretreated with BBE for 0, 12 and 24 $\mathrm{h}$ followed by stimulation with LPS $(1 \mu \mathrm{g} / \mathrm{ml})$ for $24 \mathrm{~h}$. G3PDH or GAPDH was used as internal control. Cells were harvested and subjected to RT-PCR and Western blotting as described details in materials and methods

\section{BBE reduced intracellular ROS production}

LPS triggers the inflammatory responses are known to induce the production of ROS (Palsson et al., 2004), so we measured the intracellular ROS in BV-2 cells treated with LPS with or without BBE preincubation. We used the probe $\mathrm{CMH}_{2}$ DCFDA. The intracellular ROS level was increased by LPS stimulation (89.92\%) over the level in the control cells. BBE treatment significantly reduced the LPS stimulated ROS levels. Lower levels of ROS were significantly observed in cells preincubated with BBE for $12 \mathrm{~h}(31.68 \%)$ (Figure 2). BBE even reduced the ROS levels in untreated control cells. 

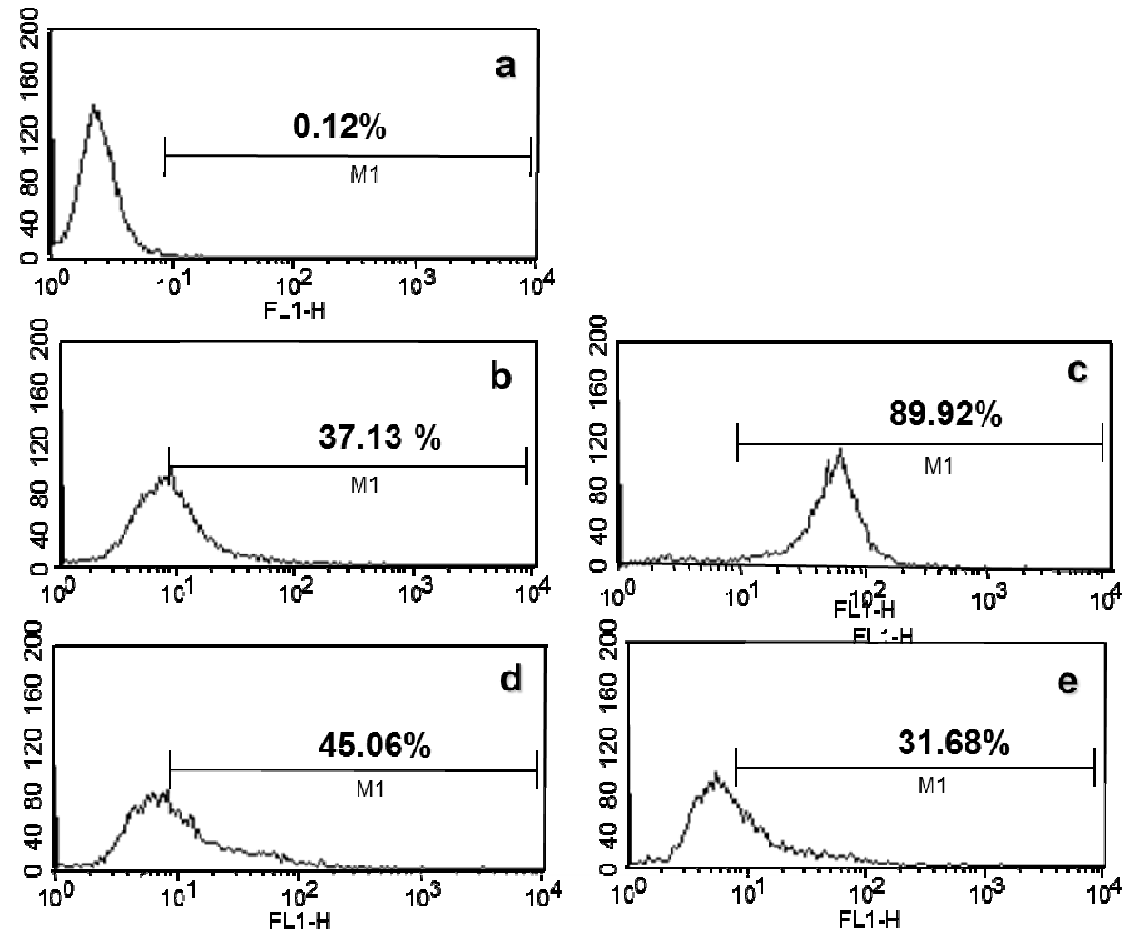

Figure 2. Effects of BBE on ROS production. Cells pretreated with BBE followed by activation of LPS for $18 \mathrm{~h}$. Evaluation of ROS in control (untreated) and treated BV-2 cells were evaluated by flow cytometer. Panel a, untreated (control) cells without dye. Panel b, cells untreated control with dye. Panel c, cells treated with LPS. Panel d, cells treated with BBE. Panel e, cells pretreated with BBE (12h) followed by LPS treatment. The percentage value given for each histogram indicates the ROS level.

\section{BBE protects the cells from LPS activation induced cell death}

To determine cell death induced by LPS, control and treated cells with BBE and LPS were stained with Hoechst 33342 and PI, and then analyzed by fluorescence microscopy. Hoechst 33342 can readily pass through all cell membranes, while PI can penetrate into necrotic or late apoptotic cells but not viable or early apoptotic cells. Both Hoechst 33342 and PI bind to DNA, and fluorescence blue and pink, respectively. Therefore, dead cells look pink and round nuclei under fluorescent microscope (Figure 3). The cells with BBE pre incubation were mostly viable (less number of PI+ cells) (Figure 3f), while LPS treated cells comprised a mixture of necrotic and apoptotic cells (Figure $3 b$ ).

We found BBE inhibited the LPS induced expression of iNOS and PrxI and the inhibitory property of BBE was found more effective in cells pretreated with BBE for $12 \mathrm{~h}$ (Figure 1). Our results were partly similar with the findings of Lau et al. (2007). LPS triggers the inflammatory responses are known to induce the production of ROS. Flow cytometric analysis showed that BBE treatment significantly reduced oxidative stress in BV-2 microglial cells through the reduction of intracellular ROS production during LPS activation. Lower levels of ROS were significantly observed in cells preincubated with BBE for $12 \mathrm{~h}$ (Figure 2). Elevated ROS generated by LPS, participates in an inflammatory process and subsequently causes the death of various cell types, including microglia. We found BBE treatment significantly reduced cell death of BV-2 microglial cells. Suk et al. (2003) showed plant flavonoids baicalein have a modest protective effect against LPS-induced microglial cell death. 

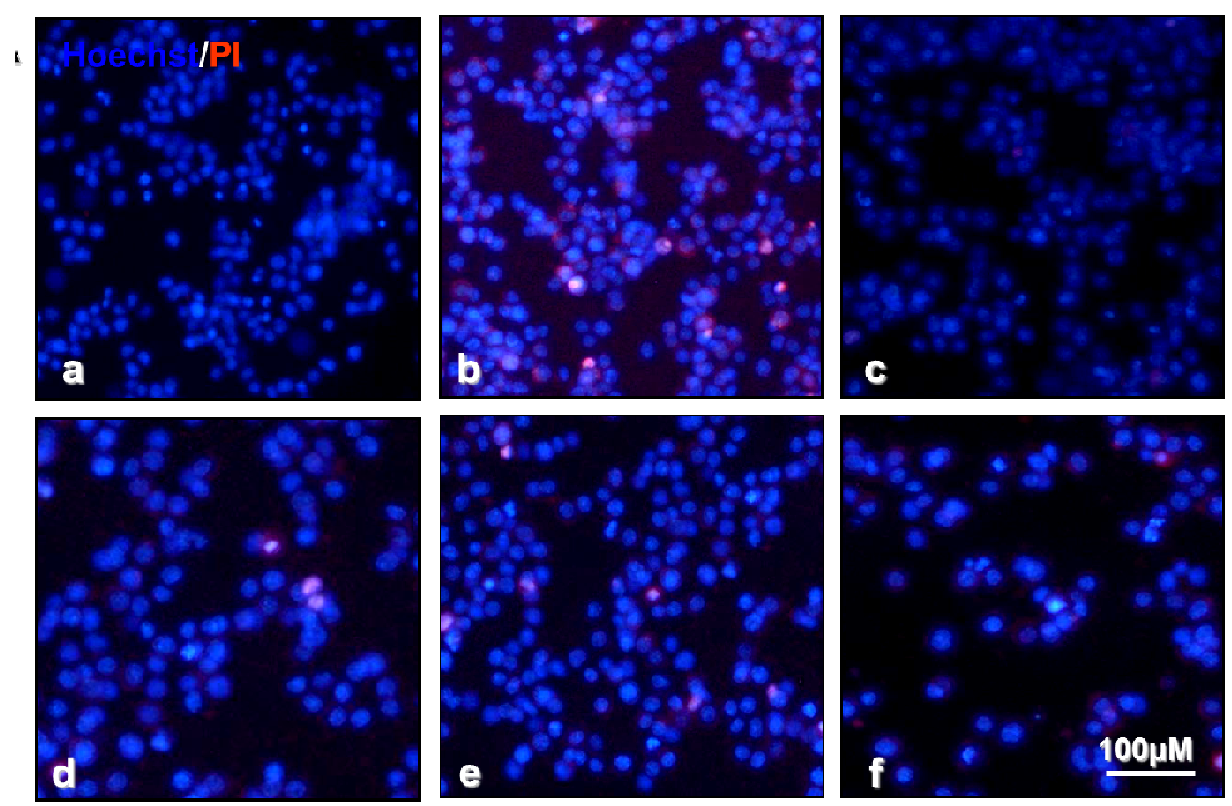

Figure 3. Effects of LPS and BBE on cells viability. BV-2 cells were treated with $1 \mu \mathrm{g} / \mathrm{ml}$ of LPS for $24 \mathrm{~h}$ with 0 , 1 and $12 \mathrm{~h}$ preincubation of BBE. Cells were stained with Hoechst (blue color) and PI (red color) represents live and dead cells, respectively. Panel a- untreated control; Panel b- cells treated with LPS; Panel c- cells treated with BBE only; Panel d, e, f cells pretreated with BBE for 0,1 and $12 \mathrm{~h}$, respectively followed by LPS treatment.

Flavonoids rich berries like blue berry, cranberry, fruits and vegetables have shown anti-inflammatory and antioxidant properties in a variety of models (Robak and Gryglewski, 1988; Middleton et al., 2000; Kim et al., 2004) and have the potential ability to limit the development and severity of certain cancers and vascular diseases including atherosclerosis, ischemic stroke, and neurodegenerative diseases of aging (Neto, 2007). Oral administration of blueberry inhibits angiogenic tumor growth and enhances the survival of mice with endothelial cell neoplasm (Gordillo et al., 2009). Cherry extracts reported to have antioxidative and anticancer activity (Lee at al., 2007). Results from our current study showed whole BBE reduces the PrxI expressions along with iNOS and protects the cell from LPS activation induced cell death. PrxI expression is highly dependent on the degree of microglial activation, which is critical for neuroinflammation caused by various stresses. So results of our current study gives scientific evidence suggesting that BBE protects cells by reducing the expressions of stressed genesiNOS and PrxI and exhibits potent anti-inflammatory and antioxidant properties.

\section{REFERENCES}

1. Davalos D, Grutzendler J, Yang G, Kim JV, Zuo Y, Jung S, Littman DR, Dustin ML, and Gan WB (2005). ATP mediates rapid microglial response to local brain injury in vivo. Nature Neuroscience 8:752758.

2. Garden GA, Moller T (2006). Microglia biology in health and disease. Journal of Neuroimmune Pharmacology 1:127-137.

3. Gordillo G, Fang H, Khanna S, Harper J, Phillips G and Sen CK (2009). Oral administration of blueberry inhibits angiogenic tumor growth and enhances survival of mice with endothelial cell neoplasm. Antioxidant and Redox Signaling 11:47-58.

4. Halliwell B (1994). Free radicals, antioxidants, and human disease: curiosity, cause, or consequence? Lancet 344:721-724. 


\section{A. Miah and others}

5. Joseph JA, Shukitt-Hale B, and Casadesus G (2005). Reversing the deleterious effects of aging on neuronal communication and behavior: beneficial properties of fruit polyphenolic compounds. American Journal of Clinical Nutrition 81(Suppl.):313S-316S.

6. Kim HP, Son KH, Chang HW, and Kang SS (2004). Anti-inflammatory plant flavonoids and cellular action mechanisms. Journal of Pharmacological Science 96:229-245.

7. Kim SU, Hwang CN, Sun HN, Jin MH, Han YH, Lee H, Kim JM, Kim SK, Yu DY, Lee DH, and Lee SH (2008). Peroxiredoxin 1 is an indicator of microglia activation and protects against hydrogen peroxide mediated microglial death. Biological \& Pharmaceutical Bulletin 31:820-825.

8. Lau FC, Donna FB, and James AJ (2007). Inhibitory effects of blueberry extract on the production of inflammatory mediators in lipopolysaccharide-activated BV-2 microglia. Journal of Neuroscience Research 85:1010-1017.

9. Lee BB, Cha MR, Kim SY, Park E, Park HR and Lee SC (2007).Antioxidative and anticancer activities of extracts of Cherry (Prunus serrulata var. spontanea) Blossoms. Plant Foods for Human Nutrition 62:79-84.

10. Middleton E Jr, Kandaswami C, and Theoharides TC (2000). The effects of plant flavonoids on mammalian cells: implications for inflammation, heart disease, and cancer. Pharmacological Review 52:673-751.

11. Neto CC (2007). Cranberry and blueberry: evidence for protective effects against cancer and vascular diseases. Molecular Nutrition \& Food Research 51(6): 652-664

12. Nimmerjahn A, Kirchhoff $\mathrm{F}$ and Helmchen $\mathrm{F}$ (2005). Resting microglial cells are highly dynamic surveillants of brain parenchyma in vivo. Science 308:1314-1318.

13. Palsson-McDermott EM and O'Neill LA (2004). Signal transduction by the lipopolysaccharide receptor, Toll-like receptor-4 [review]. Immunology 113:153-162.

14. Prior RL and Cao G (2000). Analysis of botanicals and dietary supplements for antioxidant capacity: a review. Journal of AOAC International 83:950-956.

15. Reis K, Halldin J, Fernaeus S, Pettersson C, and Land T (2006). NADPH oxidase inhibitor diphenyliodonium abolishes lipopolysaccharide induced down regulation of transferrin receptor expressions in N2a and BV-2 cell. Journal of Neuroscience Research 84:1047-1052.

16. Rice-Evans CA and Miller NJ (1996). Antioxidant activities of flavonoids as bioactive components of food. Biochemical Society Transactions 24:790-794.

17. Robak J and Gryglewski RJ (1988). Flavonoids are scavengers of superoxide anions. Biochemical Pharmacology 37:837-841.

18. Roy AM, Baliga MS and Katiyar SK (2005). Epigallocatechin-3-gallate induces apoptosis in estrogen receptor-negative human breast carcinoma cells via modulation in protein expression of p53 and Bax and caspase-3 activation. Molecular Cancer Therapeutics 4: 81-90.

19. Suk K, Lee H, Kang SS, Cho GJ, and Choi WS (2003). Flavonoid baicalein attenuates activation -induced cell death of brain microglia. Journal of Pharmacology and Experimental Therapeutics 305:638-645.

20. Yi W, Fischer J, Krewer G, Akoh CC (2005). Phenolic compounds from blueberries can inhibit colon cancer cell proliferation and induce apoptosis. Journal Agricultural and Food Chemistry 53:7320-7329. 